\title{
An upper limit on the sulphur abundance in HE 1327-2326
}

\author{
P. Bonifacio ${ }^{1}$, E. Caffau ${ }^{2,1, \star \star}$, K. A. Venn ${ }^{3}$, and D. L. Lambert ${ }^{4}$ \\ ${ }^{1}$ GEPI, Observatoire de Paris, CNRS, Univ. Paris Diderot, Place Jules Janssen, 92190 Meudon, France \\ e-mail: Piercarlo.Bonifacio@obspm. fr \\ 2 Zentrum für Astronomie der Universität Heidelberg, Landessternwarte, Königstuhl 12, 69117 Heidelberg, Germany \\ 3 Department of Physics \& Astronomy, University of Victoria, Elliott Building, Victoria, BC V8P 5C2, Canada \\ 4 The W. J. McDonald Observatory, The University of Texas at Austin, Austin, TX 78712-0259, USA
}

Received 4 May 2012 / Accepted 28 June 2012

\begin{abstract}
Context. Star HE 1327-2326 is a unique object, with the lowest measured iron abundance $([\mathrm{Fe} / \mathrm{H}] \sim-6)$ and a peculiar chemical composition that includes large overabundances of $\mathrm{C}, \mathrm{N}$, and $\mathrm{O}$ with respect to iron. One important question is whether the chemical abundances in this star reflect the chemical composition of the gas cloud from which it was formed or if they have been severely affected by other processes, such as dust-gas winnowing.

Aims. We measure or provide an upper limit to the abundance of the volatile element sulphur, which can help to discriminate between the two scenarios.

Methods. We observed HE 1327-2326 with the high resolution infra-red spectrograph CRIRES at the VLT to observe the S I lines of Multiplet 3 at $1045 \mathrm{~nm}$.

Results. We do not detect the $\mathrm{S}$ I line. A $3 \sigma$ upper limit on the equivalent width (EW) of any line in our spectrum is $E W<0.66 \mathrm{pm}$. Using either one-dimensional static or three-dimensional hydrodynamical model-atmospheres, this translates into a robust upper limit of $[\mathrm{S} / \mathrm{H}]<-2.6$.

Conclusions. This upper limit does not provide conclusive evidence for or against dust-gas winnowing, and the evidence coming from other elements (e.g., $\mathrm{Na}$ and $\mathrm{Ti}$ ) is also inconclusive or contradictory. The formation of dust in the atmosphere versus an origin of the metals in a metal-poor supernova with extensive "fall-back" are not mutually exclusive. It is possible that dust formation distorts the peculiar abundance pattern created by a supernova with fall-back, thus the abundance ratios in HE 1327-2326 may be used to constrain the properties of the supernova(e) that produced its metals, but with some caution.
\end{abstract}

Key words. stars: abundances - stars: individual: HE 1327-2326 - stars: Population II - Galaxy: evolution - Galaxy: abundances line: formation

\section{Introduction}

In the widely accepted cosmological picture, the Universe experienced a very hot and dense phase (big bang) during which only the lightest nuclei were synthesised. All the nuclei, from $\mathrm{C}$ to $\mathrm{U}$, were manufactured by stars in the subsequent evolution of the Universe. A corollary of this scenario is that the most pristine stars were formed with a very low content of heavy elements, if any at all. Low mass stars have very long lifetimes, in fact stars with masses less than $0.8 M_{\odot}$ have main sequence lifetimes that exceed the age of the Universe, as derived from the measurement of the Hubble constant (Freedman et al. 2001). According to the standard theory of stellar evolution (see Pinsonneault 1997, for a review on mixing in stars) very minor changes are expected in the chemical composition of the atmosphere of a low mass star during its main sequence lifetime. Thus such stars hold the memory of the chemical composition of the interstellar medium from which they formed. For this reason, stars with extremely low metallicity are actively sought by many research groups (an incomplete list includes Beers et al. 1985, 1992; Cayrel et al. 2004; Christlieb et al. 2008; Cohen et al. 2008; Lai et al. 2008; Bonifacio et al. 2009; Caffau et al. 2011b; Bonifacio et al. 2012)

* Based on spectra obtained with CRIRES at the $8.2 \mathrm{~m}$ Antu ESO telescope, programme 386.D-0095.

$\star \star$ Gliese Fellow. to address the question of whether low mass stars of primordial (i.e. only $\mathrm{H}, \mathrm{He}$, and $\mathrm{Li}$ ) chemical composition exist or not (see Bromm \& Larson 2004, for a review on star formation in primordial stars). In the course of the very successful HamburgESO Survey (Christlieb et al. 2008) the two stars with the lowest $[\mathrm{Fe} / \mathrm{H}]$ content so-far measured were discovered: HE 0107-5240 (Christlieb et al. 2002), a red giant (Christlieb et al. 2004), and HE 1327-2326 (Frebel et al. 2005), a subgiant (Korn et al. 2009). These two stars show a very remarkable chemical composition in which carbon, nitrogen, and oxygen are enhanced by several orders of magnitude over iron and other iron-peak elements. These large overabundances persist even when the analysis uses 3D hydrodynamical models that imply large downward revisions of the abundances derived from molecular lines (Collet et al. 2006; Frebel et al. 2008). In turn, this implies that the global metallicity $Z$ of these stars is not as extreme as implied by their iron abundances, e.g., $\log \left(Z / Z_{\odot}\right) \approx-2.5$ for HE 1327-2326. This situation is at odds with what is observed in other extremely metal-poor stars (see e.g. Cayrel et al. 2004; Bonifacio et al. 2009; and Bonifacio 2010, for a review), that show no enhancement of $\mathrm{C}$, an underabundance of $\mathrm{N}$, and a mild enhancement of $\mathrm{O}$ (relative to $\mathrm{Fe}$ ). This exceptional situation stimulated a theory (Bromm \& Loeb 2003) that actually requires such an exceptional chemical composition for low-mass star formation at extremely low metallicities, i.e., where fine structure lines of $\mathrm{O}_{\mathrm{I}}$ and $\mathrm{C}$ II provide the cooling necessary to allow the collapse of a 
gas cloud of low mass. The discovery of SDSS J102915+172927 (Caffau et al. 2011c, 2012), with $[\mathrm{Fe} / \mathrm{H}] \sim-5$ and no measurable enhancement of $\mathrm{C}$ or $\mathrm{N}$, lies in the "forbidden zone" of the Bromm \& Loeb (2003) theory, and casts doubt on the necessity of a peculiar chemical composition at low metallicity. Other means of forming low mass stars, that rely on fragmentation, either through dust (Schneider et al. 2003; Salvadori et al. 2007; Schneider et al. 2012a; Klessen et al. 2012; Schneider et al. 2012b), or $\mathrm{H}_{2}$ cooling (Clark et al. 2011; Greif et al. 2011), appear viable as alternative routes to star formation and can explain the existence of stars such as SDSS J102915+172927.

The peculiar chemical composition of HE 0107-5240 and HE 1327-2326 may arise in several ways, either through enrichment by at least two supernovae (Bonifacio et al. 2003; Limongi et al. 2003) or through hypernovae (Umeda \& Nomoto 2003; Iwamoto et al. 2005).

Is the chemical composition of HE0107-5240 and HE 1327-2326 so exceptional and confined to the extremely metal-poor stars? In an intriguing paper, Venn \& Lambert (2008) pointed out that the chemical composition pattern of these two stars, when plotted as a function of condensation temperature, shows similarities to that of post-AGB and $\lambda$ Boo stars. Both kinds of objects can be interpreted as the result of underabundances of elements which condense onto dust most readily, i.e., at the highest temperatures, a process dubbed "dust-gas winnowing". In such a scenario, HE0107-5240 and HE 1327-2326 could be simply stars affected by dust-gas winnowing rather than extremely metal-poor stars. One way to verify such a scenario is to measure the abundance of volatile elements such as S or $\mathrm{Zn}$. If the extreme iron deficiency of HE 0107-5240 and HE 1327-2326 is due to dust, we can expect the abundance of the volatile elements to track more closely that of oxygen, rather than that of iron.

This paper reports our attempt to measure, or derive a meaningful upper limit, to the abundance of $\mathrm{S}$ in the brighter of the two stars: HE 1327-2326. For Zn, there are already upper limits from Aoki et al. (2006) $([\mathrm{Zn} / \mathrm{Fe}]<3.07)$ using HDS-Subaru spectra and Frebel et al. (2008) ([Zn/Fe] < 3.01) using UVES-VLT spectra, which are too high to examine the claim of dust-gas winnowing, and it appears difficult to lower those with existing instrumentation. The available lines of $\mathrm{S}$ are stronger than those of $\mathrm{Zn}$ making the prospect of a detection more favourable. Previously existing data covered the $\mathrm{S}_{\text {I }}$ lines of multiplet 1 around $920 \mathrm{~nm}$, however, that whole region is severely affected by telluric absorption lines so that no meaningful upper limit could be derived. In this paper, we focus on the lines of multiplet 3 around $1045 \mathrm{~nm}$. Such lines lie in a region that is fairly free from telluric absorption and can be usefully used to derive sulphur abundances (Caffau et al. 2007, 2010).

\section{Observations and results}

CRIRES (Kaeufl et al. 2004) at the ESO VLT Antu $8.2 \mathrm{~m}$ telescope was used to acquire high resolution spectra of the region around the $S_{\text {I }}$ multiplet 3 . The standard setting in order 54 centred at $1045.9 \mathrm{~nm}$ with a slit of $0 . ' 4$ providing a resolving power $R \sim 50000$ was used. Since the star is rather faint $(J=12.36)$ we used long detector integration times (DITs) of $300 \mathrm{~s}, 6$ exposures totalled 4 such DITs, and one exposure totalled 6 DITs. The observations were carried out in service mode using the standard on-slit nodding (throw of $10^{\prime \prime}$ ) to acquire the necessary sky exposures for sky subtraction. The spectra themselves, as well as full

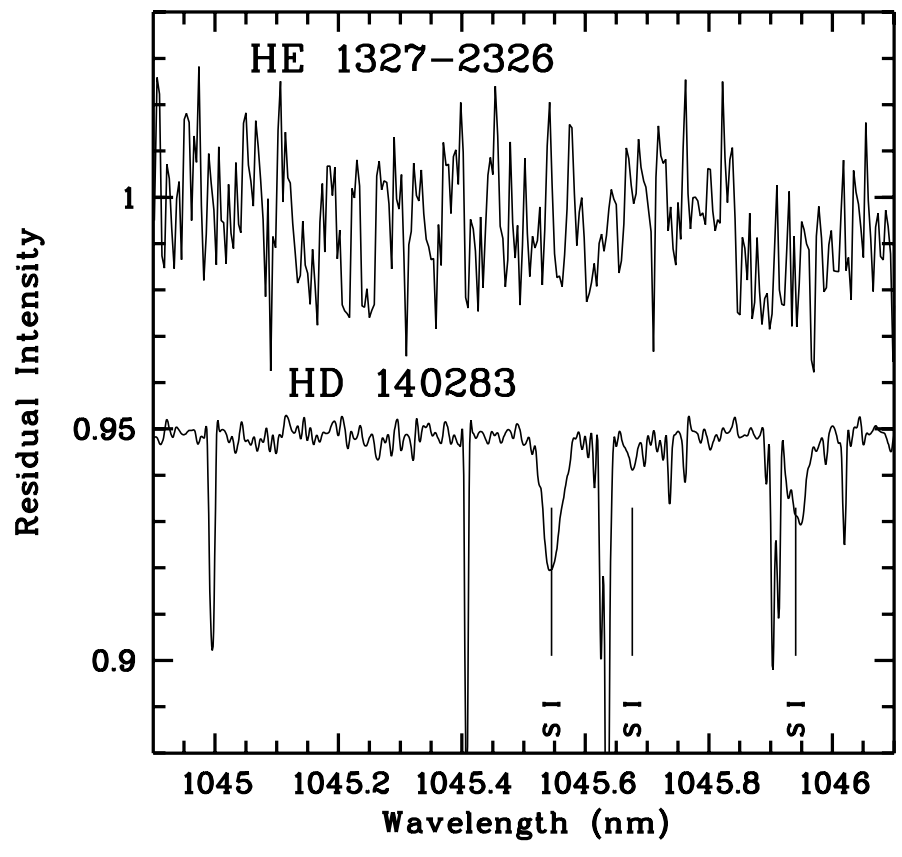

Fig. 1. The combined CRIRES spectrum of HE 1327-2326 in the spectral region of the $S_{\text {I }}$ Mult. 3 lines. The spectrum has been shifted to zero radial velocity assuming $v_{r}=63.8 \mathrm{~km} \mathrm{~s}^{-1}$ (Aoki et al. 2006). Below, shifted by 0.05 units in the vertical axis, the CRIRES spectrum of HD 140283 (Caffau et al. 2010) in which all three lines of Mult. 3 are clearly detected, the strongest line has an equivalent width of about $1.0 \mathrm{pm}$, only slightly larger than the upper limit we determined for HE 1327-2326.

details on the observations are available from the ESO archive $^{1}$. The Adaptive Optic correction was done on-axis on the target itself. The reduced spectra, using the CRIRES pipeline, were provided by ESO in the PI-package. Each spectrum was shifted to wavelength in air, and corrected by the barycentric correction at the time of observation. It was then rebinned at a constant step of $0.004 \mathrm{~nm}$ and the median of the seven spectra was computed. The signal-to-noise ratio of the combined spectrum is about 67.

The combined normalised spectrum has been shifted to the rest wavelength, assuming a radial velocity of $63.8 \mathrm{~km} \mathrm{~s}^{-1}$ (Aoki et al. 2006), and is shown in Fig. 1. No S lines nor telluric lines are apparent. Using the Cayrel formula (Cayrel 1988), a $3 \sigma$ detection upper limit for this spectrum is $0.66 \mathrm{pm}$. Attributing this to the strongest line of the $\mathrm{S}$ multiplet, we derive an upper limit to the the sulphur abundance in HE 1327-2326 using a model atmospheres analysis. The atmospheric parameters for this star are taken from Frebel et al. (2008); $T_{\text {eff }}=6180 \mathrm{~K}, \log g=3.7$ (cgs units), and microturbulence of $1.6 \mathrm{~km} \mathrm{~s}^{-1}$. The ATLAS 12 code (Kurucz 2005; but see also Castelli 2005) was used to compute a model atmosphere. For this model, the chemical composition found by (Frebel et al. 2008) was adopted, also where the abundances of elements not previously observed were scaled by -5.5 with respect to the solar value, or -5.1 for the $\alpha$ elements following the general trend among metal-poor stars. For the solar abundance of $\mathrm{S}$ we have assumed $A(\mathrm{~S})=7.16$ (Caffau et al. 2011a). We also have two hydrodynamical simulations, computed with CO ${ }^{5}$ BOLD (Freytag et al. 2002, 2003; Wedemeyer et al. 2004; Freytag et al. 2012), and their associated 1D LHD models (Caffau \& Ludwig 2007), although their chemical composition is standard. We used the atomic data provided

\footnotetext{
1 http://archive.eso.org/wdb/wdb/eso/sched_rep_arc/ query?progid=386.D-0095 (A)
} 
Table 1. Atomic parameters of the sulphur lines, oscillator strengths from Podobedova et al. (2009).

\begin{tabular}{lccc}
\hline \hline $\begin{array}{l}\text { Wavelength } \\
(\mathrm{nm}) \text { air }\end{array}$ & Transition & $\log g f$ & $\begin{array}{c}\chi_{\mathrm{lo}} \\
(\mathrm{eV})\end{array}$ \\
\hline 1045.5449 & ${ }^{3} \mathrm{~S}_{1}^{\mathrm{o}}-{ }^{3} \mathrm{P}_{2}$ & 0.25 & 6.86 \\
1045.6757 & ${ }^{3} \mathrm{~S}_{1}^{\mathrm{o}}-{ }^{3} \mathrm{P}_{0}$ & -0.45 & 6.86 \\
1045.9406 & ${ }^{3} \mathrm{~S}_{1}^{\mathrm{o}}-{ }^{3} \mathrm{P}_{1}$ & 0.03 & 6.86 \\
\hline
\end{tabular}

Table 2. Upper limits on the S abundance in HE 1327-2326.

\begin{tabular}{lccccc}
\hline \hline Model & $T_{\text {eff }}$ & $\log g$ & {$[\mathrm{M} / \mathrm{H}]$} & $A(\mathrm{~S})$ & NLTE corr \\
\hline ATLAS 12 & 6180 & 3.7 & -5.5 & $<4.72$ & -0.3 \\
CO ${ }^{5}$ BOLD & 6300 & 4.0 & -3.0 & $<4.87$ & -0.3 \\
CO ${ }^{5}$ BOLD & 6300 & 3.5 & -3.0 & $<4.68$ & -0.3 \\
\hline
\end{tabular}

in Table 1 and the Linfor $3 \mathrm{D}^{2}$ code to compute the $\mathrm{S}$ abundance implied by the upper limit on the equivalent width. The results are provided in Table 2, which also includes the NLTE correction deduced from the tables of Takeda et al. (2005), assuming metallicity -3.0 (this metallicity is preferred to avoid extrapolation). Our observation provides a robust upper limit of $[\mathrm{S} / \mathrm{H}]<-2.60$, independent of the choice of model atmosphere and line formation code.

\section{Discussion}

Our upper limit to the $\mathrm{S}$ abundance, $[\mathrm{S} / \mathrm{H}]<-2.6$ or $[\mathrm{S} / \mathrm{Fe}]<3.4$, is the tightest upper limit obtainable for HE1327-2326 due to our selection of $\mathrm{S}_{\mathrm{I}}$ lines. This limit has two possible interpretations given the overall composition of this extremely iron-poor star (Frebel et al. 2008): (i) the star was formed from primordial gas contaminated by first-generation supernovae only; or (ii) the composition is severely affected by dust-gas winnowing, i.e., elements which condense into and onto dust grains at moderate temperatures are underabundant with respect to their initial stellar abundances.

Frebel et al. (2008, Fig. 17) summarise the predicted abundances from several models for first-generation supernovae. Several of these models provide a reasonable account of the observed abundances of $\mathrm{C}, \mathrm{N}, \mathrm{O}, \mathrm{Na}, \mathrm{Mg}, \mathrm{Al}$, and $\mathrm{Ca}$ to $\mathrm{Ni}$. In the case of $\mathrm{S}$, the three models providing a prediction give $[\mathrm{S} / \mathrm{Fe}]$ from 1.9 to 0.9 which are, of course, consistent with our observed limit $[\mathrm{S} / \mathrm{Fe}]<3.4$. Given the nature of nucleosynthesis by supernovae from massive stars, the $[\mathrm{S} / \mathrm{Fe}]$ seems certain to be bracketed by the observed $[\mathrm{Mg} / \mathrm{Fe}]$ and $[\mathrm{Ca} / \mathrm{Fe}]$, i.e., 2 to 1 in this star. Other measurements of $\mathrm{S}$ in stars are not particularly helpful because they do not extend to ultra-metal poor stars; for example, $[\mathrm{S} / \mathrm{Fe}] \simeq+0.3$ for metal-poor stars down to $[\mathrm{Fe} / \mathrm{H}] \simeq-3.5$ (Nissen et al. 2007; Spite et al. 2011). In summary, the limit $[\mathrm{S} / \mathrm{Fe}]<3.4$ is too loose a value to contradict the suggestion that HE 1327-2326 formed from gas contaminated by first-generation supernovae.

According to the radical alternative viewpoint, HE 13272326's composition may be seriously affected by dust-gas winnowing. A signature of winnowing is that the underabundance of an element is correlated with its predicted condensation temperature. Elements with the lowest condensation temperatures are the

\footnotetext{
${ }^{2}$ http://www.aip.de/ mst/Linfor3D/linfor_3D_manual.pdf
}

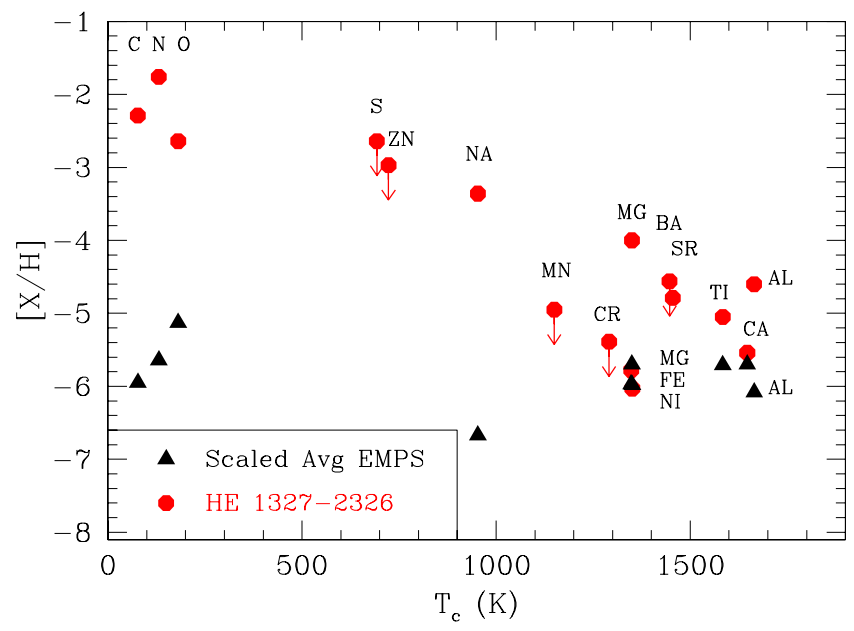

Fig. 2. Abundances in HE 1327-2326 (red circles) as a function of condensation temperature (Lodders 2003) compared to those of the "average star" (black triangles), as defined by Limongi \& Chieffi (2012) starting from the data of Cayrel et al. (2004) and Spite et al. (2005). The symbols for $\mathrm{Fe}$ and $\mathrm{Ni}$ in the "average star" are on top of one another.

surest indicators of a star's initial metallicity. In this regard, C, N, and $\mathrm{O}$ suggest a metallicity much greater than the $[\mathrm{Fe} / \mathrm{H}]=-6$ obtained from a set of $\mathrm{Fe}$ I lines.

In Fig. 2, we plot the abundances of HE 1327-2326 as a function of condensation temperature $T_{\mathrm{C}}$ predicted by Lodders (2003) for the solar composition at low gas pressure, and compare it to that of the "average" metal-poor star. This is defined by Limongi \& Chieffi (2012), starting from the data of Cayrel et al. (2004) and Spite et al. (2005). The sample of Cayrel et al. (2004) spans the metallicity range -2.5 to -4.0 . The fact that the pattern of this "average star" closely follows that of SDSS J102915+172927 (Caffau et al. 2011c, 2012) implies that this pattern extends down to $[\mathrm{Fe} / \mathrm{H}]=-5.0$. To make the comparison in Fig. 2, we assume that stars with this pattern exist down to $[\mathrm{Fe} / \mathrm{H}]=-6.0$. The figure highlights the fact that the abundance pattern in the "average" metal poor star shows no trend with $T_{\mathrm{C}}$, while HE 1327-2326 shows a very definite correlation. The upper limits for $\mathrm{S}$ and $\mathrm{Zn}$ fall close to the mean trend for elements with $T_{\mathrm{C}}<1000 \mathrm{~K}$.

The chosen condensation temperatures were computed for a solar $\mathrm{C} / \mathrm{O}$ ratio (i.e., 0.5) but the observed $\mathrm{C} / \mathrm{O}$ is non-solar (i.e., 2.3) - a change which will require adjustments to the $T_{\mathrm{C}}$ values. It would be helpful to calculate $T_{\mathrm{C}}$ for a range of compositions more representative of the possible initial compositions of HE 1327-2326. This may result in a smoother trend of abundances versus $T_{\mathrm{C}}$. In a different context, an attempt to derive chemical compositions of planets hosted by stars of different chemical composition, Bond et al. (2010) have computed the $T_{\mathrm{C}}$ per element for the chemical compositions of different planet host stars. The differences in the $\mathrm{C} / \mathrm{O}$ ratios can be quite significant, e.g., $300 \mathrm{~K}$. Although this result is unlikely to apply directly to the low metallicity star HE 1327-2326, it suggests that significant variations due to chemical composition are possible.

In Fig. 3 we plot $[\mathrm{X} / \mathrm{H}]$ of HE 1327-2326 versus the condensation temperature $T_{\mathrm{C}}$, and in the same plot are shown the abundances of several comparison stars that are known to show the effects of dust-gas winnowing: post-AGB and RV Tauri stars. These post-AGB and RV Tauri stars show IR excesses as evidence of warm dust (Lamers 1986; Waelkens et al. 1991; Gielen et al. 2008; Friedemann et al. 1996; de Ruyter et al. 2005). In spite of the general resemblance in the chemical pattern, 


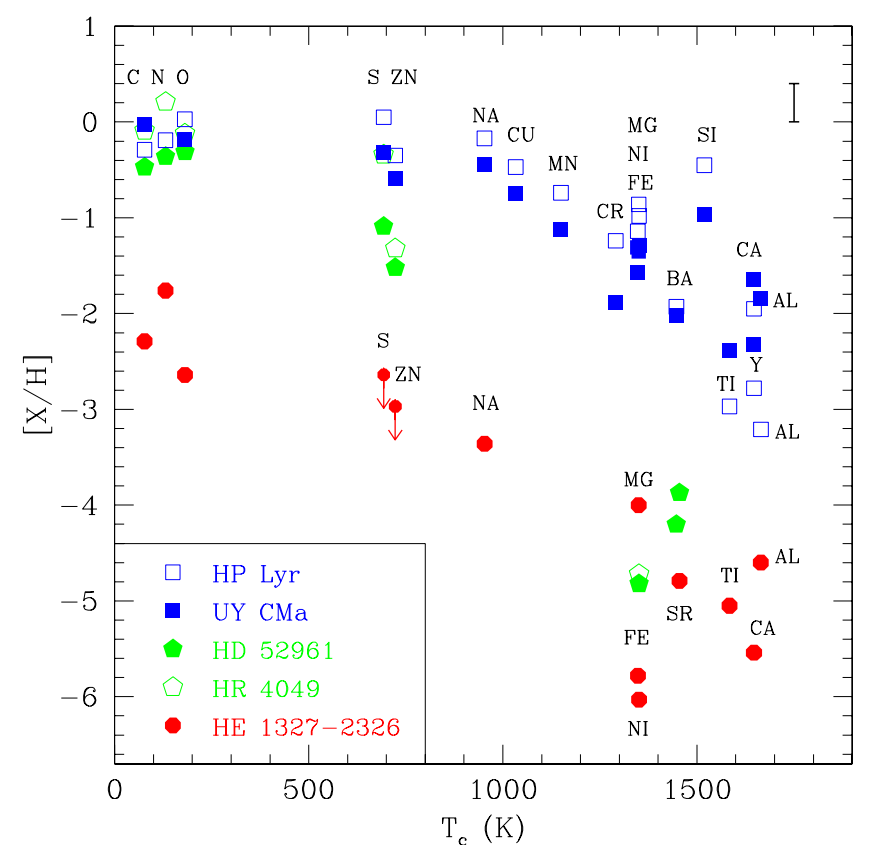

Fig. 3. Elemental abundances for HE 1327-2326, two post-AGB stars, and RV Tauri stars plotted versus condensation temperature $T_{\mathrm{C}}$ from Lodders (2003) for the solar composition. HP Lyr and UY CMa are RV Tauri stars, the abundances are from Giridhar et al. (2005). HD 52961 and HR 4049 are A-type post-AGB stars and the abundances are from Waelkens et al. (1991), plus Zn from Takeda et al. (2002), and Van Winckel et al. (1992), respectively. This is a slightly modified version of Fig. 2 of Venn \& Lambert (2008).

HE 1327-2326 shows considerable scatter among the C, N, and $\mathrm{O}$ abundances at low $T_{\mathrm{C}}$ and again at high $T_{\mathrm{C}}$, e.g., $\mathrm{Mg}$ relative to $\mathrm{Fe}$ and $\mathrm{Ni}$.

In Fig. 4 we plot the abundances as a function of $T_{\mathrm{C}}$ for HE 1327-2326 together with those of the RV Tauri star HP Lyr scaled by -2 dex. The mismatch of $\mathrm{Mg}$ with $\mathrm{Fe}$ and $\mathrm{Ni}$ is obvious. What is also striking is that only a subset of the heavier elements $(A>22$ shown as open symbols) show large deviations from the scaled pattern of HP Lyr, i.e., only the iron-group elements ( $\mathrm{Fe}$ and $\mathrm{Ni}$, and the upper limits on $\mathrm{Cr}$ and $\mathrm{Mn}$ ) differ from the dust-gas winnowing pattern. The other heavy elements ( $\mathrm{Sr}$, and the upper limits on $\mathrm{Ba}$ and $\mathrm{Zn}$ ) are consistent with pattern in HP Lyr.

A similar exercise is shown in Fig. 5 where the abundances of HP Lyr and the post-AGB star HD 52961 are compared. This figure suggests, as do studies of RV Tauri variables, that dustgas winnowing does not result in a universal abundance pattern with $T_{\mathrm{C}}$. The properties of a debris disk would depend on composition, age, distance from the host star, and the dust temperature. One should however also be aware that we are comparing the patterns of stars that occupy different positions in the Hertzsprung-Russel diagram. In particular the mass of the outer convection zone should be revelant to the final abundance pattern observable in the photosphere in the presence of dust-gas winnowing.

A complication is that we do not know what the "intrinsic" abundances in HE 1327-2326 would be before distortion by dustgas winnowing if this star has been affected by the presence of dust. While it is tempting to assign the $[\mathrm{O} / \mathrm{H}]$ value as the intrinsic metallicity (i.e., $[\mathrm{X} / \mathrm{H}]=-2$ ), this is complicated by the type(s) of SNe that would have enriched the proto stellar nebula. If the abundance pattern of HE 1327-2326 is a signature of

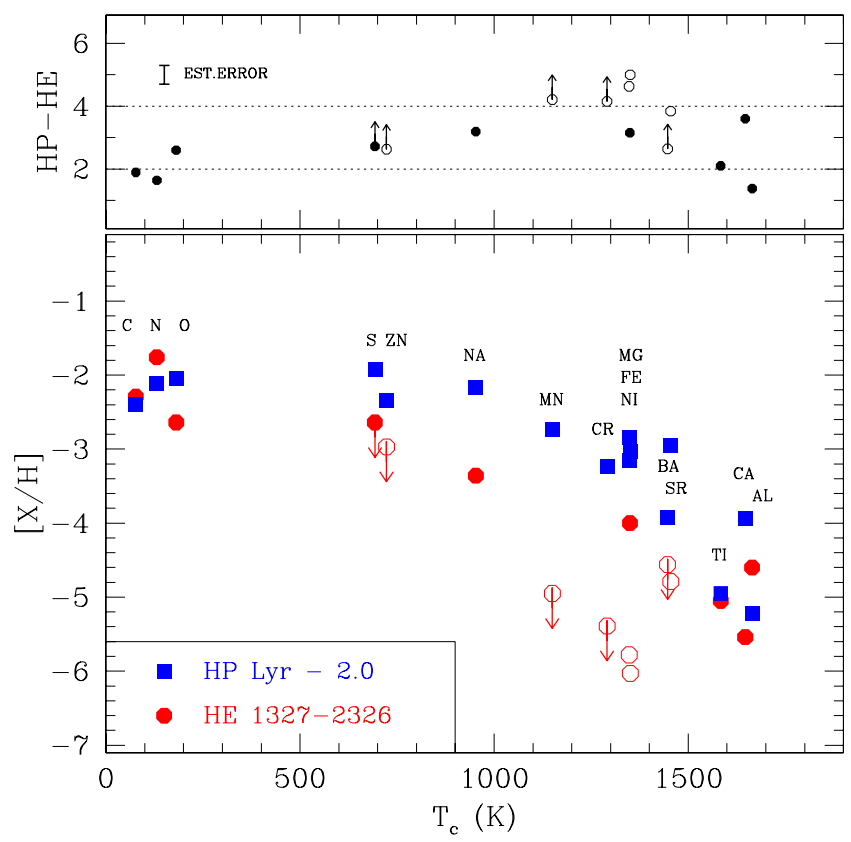

Fig. 4. Abundances in HE 1327-2326 compared to those of the RV Tauri star HP Lyr, after the latter have been scaled by -2 dex. For HE 1327-2326 filled symbols are used for elements with $A \leq 22$ and open symbols the heavier elements. The upper box shows the differences in the abundances scaled HP Lyr - HE 1327-2326.

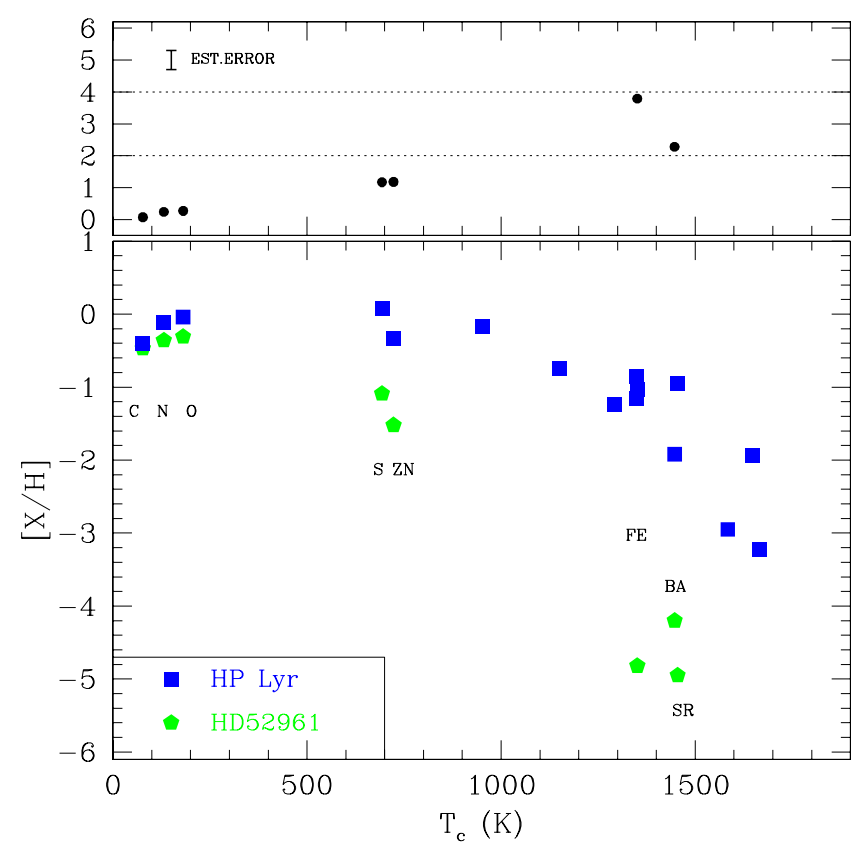

Fig. 5. Abundances in the post-AGB star HD 52961 compared to those in the RV Tauri star HP Lyr. The upper box shows the differences in the abundances HP Lyr - HD 52961.

nucleosynthesis in the supernovae of the earlier generation, it seems unavoidable to invoke "fall-back" in at least one of the supernovae, in order to account for the very high $\mathrm{O} / \mathrm{Fe}$ ratio. This is the case also for HE 0107-5240 (Bonifacio et al. 2003; Limongi et al. 2003; Umeda \& Nomoto 2003; Iwamoto et al. 2005). The "fall-back" increases when the core is more compact owing to a stronger reverse shock. This tends to occur in the Pop III models that result in very compact black holes, whereas the Pop I (solar metallicity) models do not (Zhang et al. 2008), although there are 
other factors to consider such as explosion energy, rotation rate, and progenitor mass (Joggerst et al. 2010). Thus, the specific role of metallicity and thus Pop I/III progenitor is unclear, e.g., Moriya et al. (2010) have shown that their solar metallicity corecollapse supernovae models can result in significant "fall-back", used to explain the light curve and lack of metal absorption lines in the spectrum of the peculiar supernova SN 2008ha.

Referring to Fig. 17 of Frebel et al. (2008), the general trend with atomic number of the model prediction is by and large similar to the observed one. Unfortunately the pattern produced by "fall-back" is also similar to what would be the effect of dust formation. The exceptions are $\mathrm{Na}, \mathrm{S}$, and Ti. Sodium is only moderately depleted onto dust and is in fact found at the same level as CNO and S for the scaled abundances of HP Lyr in Fig. 4. In metal-poor stars $\mathrm{Na}$ is found to be slightly underabundant with respect to iron, 0.2-0.3 dex (Andrievsky et al. 2007). On the other hand all the $\mathrm{SN}$ models underproduce $\mathrm{Na}$ with respect to $\mathrm{N}$ by at least 1 dex. The fact that in Fig. 4 the $\mathrm{Na}$ abundance in HE 1327-2326 is about 1 dex below the scaled abundance of HP Lyr suggests that the result is due to nucleosynthesis, not of dust formation. Sulphur is predicted to be roughly at the same level as $\mathrm{Ca}$ in the $\mathrm{SNe}$ products, but our upper limit is inconclusive, being consistent with $[\mathrm{S} / \mathrm{Ca}] \sim 0$ as expected from the $\mathrm{SN}$ nucleosynthesis and $[\mathrm{S} / \mathrm{Ca}]<0$ as expected from dust formation. Always with reference to Fig. 17 of Frebel et al. (2008), titanium is expected to be underproduced in SNe with respect to $\mathrm{Ca}$, by about 1 dex. The observed abundance of $\mathrm{Ti}$ in HE 1327-2326 is higher than all the model predictions. In Fig. 4 we may see the the Ti abundance almost matches that of HP Lyr scaled by -2.0 dex. This would therefore suggest that we are observing the effects of dust and not of nucleosynthesis.

There remains one significant difference between the comparison in Fig. 4 and that in Fig. 5. In Fig. 5 the abundances $[\mathrm{X} / \mathrm{H}]$ of the $\mathrm{CNO}$ elements, presumably unaffected by dust, are very similar. Instead in Fig. 4, the pattern is different. Possibly the most remarkable fact is that in HE 1327-2326 oxygen displays the lowest abundances, while it is the largest in HP Lyr. The pattern in $\mathrm{CNO}$ abundances found in extremely metal poor stars at $[\mathrm{Fe} / \mathrm{H}] \sim-3.5$ is $[\mathrm{O} / \mathrm{H}] \sim-2.5$ (González Hernández et al. 2010, unevolved stars), [C/H] -3.5 (Spite et al. 2005, for "unmixed" giants), and $[\mathrm{N} / \mathrm{H}] \sim-3.5$ (Spite et al. 2005, for "unmixed" giants), which is clearly different from that observed in HE 1327-2326. Therefore even if the abundances of other elements were affected by dust-gas winnowing, the abundances of $\mathrm{C}, \mathrm{N}$, and $\mathrm{O}$ are peculiar, with respect to those of other extremely metal-poor stars.

In an approach based on theoretical models of Galactic chemical evolution, we may consider adjusting to the "intrinsic" N and O abundances of Goswami \& Prantzos (2000), however this widens the gap between the $\mathrm{N}$ and $\mathrm{O}$ abundances to about 1.2 dex. At high $T_{\mathrm{C}}$ in Fig. 3, corrections for the initial abundances of the $\alpha$-elements $\mathrm{Mg}, \mathrm{Ca}$, and $\mathrm{Ti}$ would marginally reduce the spread because $[\alpha / \mathrm{Fe}] \simeq+0.4$ initially. However, Al remains a clear outlier because $[\mathrm{Al} / \mathrm{Fe}] \simeq 0$ initially (Goswami \& Prantzos 2000; Kobayashi \& Nakasato 2011).

Amongst the mechanisms that may have distorted the abundances of HE 1327-2326, one should also consider atomic diffusion. This has been considered in detail by Korn et al. (2009), who concluded, on the basis of parametric models of diffusion in the presence of turbulence, that the effects are small, of the order of 0.2 dex. Pure diffusion, not inhibited by turbulence, would produce effects much larger than this. It is not inconceivable that some peculiar phenomenon takes place in connection with atomic diffusion at the lowest metallicities, e.g., one peculiar behaviour found at the lowest metallicities is the meltdown of the Spite plateau below $[\mathrm{Fe} / \mathrm{H}]=-3$ (Sbordone et al. 2010).

\section{Conclusions}

Our upper limit to the $\mathrm{S}$ abundance does not provide decisive evidence in favour of the formation of dust in the atmosphere of HE 1327-2326, but this cannot be ruled out either. The pattern in the light element abundances as a function of condensation temperature still suggests that HE 1327-2326's atmosphere could have been distorted by dust-gas winnowing. The abundances resulting from a SN experiencing extensive "fall-back" would show a similar pattern though. Thus, interpretation of the abundance pattern is not unambiguous. Two measured elements that could help to discriminate between the two scenarios, $\mathrm{Na}$ and $\mathrm{Ti}$, provide contradictory results. $\mathrm{Na} / \mathrm{N}$ supports a nucleosynthetic origin for the abundance pattern, on the contrary $\mathrm{Ti}$ is in better agreement with the dust hypothesis. Nucleosynthesis and dustgas winnowing are not mutually exclusive (e.g., Schneider et al. 2012a), and it is possible that dust formation distorts the peculiar abundance pattern created by a supernova with extensive "fallback". The available information, albeit contradictory, suggests that any diagnostics for supernovae yields based on abundance ratios in this star be treated with caution.

Acknowledgements. We are grateful to F. Herwig, A. Heger, and M. Pignatari for useful discussions on nucleosynthesis in low metallicity supernovae. We wish to thank the referee, A. Korn, for his thoughtful comments that helped to improve the paper. We acknowledge support from the Programme National de Physique Stellaire (PNPS) and the Programme National de Cosmologie et Galaxies (PNCG) of the Institut National des Sciences de 1'Univers of CNRS. K.A.V. also thanks NSERC for support through the Discovery Grant program.

\section{References}

Andrievsky, S. M., Spite, M., Korotin, S. A., et al. 2007, A\&A, 464, 1081 Aoki, W., Frebel, A., Christlieb, N., et al. 2006, ApJ, 639, 897

Beers, T. C., Preston, G. W., \& Shectman, S. A. 1985, AJ, 90, 2089

Beers, T. C., Preston, G. W., \& Shectman, S. A. 1992, AJ, 103, 1987

Bond, J. C., O'Brien, D. P., \& Lauretta, D. S. 2010, ApJ, 715, 1050

Bonifacio, P. 2010, IAU Symp., 265, 81

Bonifacio, P., Limongi, M., \& Chieffi, A. 2003, Nature, 422, 834

Bonifacio, P., Spite, M., Cayrel, R., et al. 2009, A\&A, 501, 519

Bonifacio, P., Sbordone, L., Caffau, E., et al. 2012, A\&A, 542, A87

Bromm, V., \& Larson, R. B. 2004, ARA\&A, 42, 79

Bromm, V., \& Loeb, A. 2003, Nature, 425, 812

Caffau, E., \& Ludwig, H.-G. 2007, A\&A, 467, L11

Caffau, E., Faraggiana, R., Bonifacio, P., Ludwig, H.-G., \& Steffen, M. 2007, A\&A, 470, 699

Caffau, E., Sbordone, L., Ludwig, H.-G., Bonifacio, P., \& Spite, M. 2010, Astron. Nachr., 331, 725

Caffau, E., Ludwig, H.-G., Steffen, M., Freytag, B., \& Bonifacio, P. 2011a, Sol. Phys., 268, 255

Caffau, E., Bonifacio, P., François, P., et al. 2011b, A\&A, 534, A4

Caffau, E., Bonifacio, P., François, P., et al. 2011c, Nature, 477, 67

Caffau, E., Bonifacio, P., François, P., et al. 2012, A\&A, 542, A51

Castelli, F. 2005, Mem. Soc. Astron. It. Suppl., 8, 25

Cayrel, R. 1988, in The Impact of Very High S/N Spectroscopy on Stellar Physics, eds. G. Cayrel de Strobel, \& M. Spite, IAU Symp., 132, 345

Cayrel, R., Depagne, E., Spite, M., et al. 2004, A\&A, 416, 1117

Cohen, J. G., Christlieb, N., McWilliam, A., et al. 2008, ApJ, 672, 320

Christlieb, N., Bessell, M. S., Beers, T. C., et al. 2002, Nature, 419, 904

Christlieb, N., Gustafsson, B., Korn, A. J., et al. 2004, ApJ, 603, 708

Christlieb, N., Schörck, T., Frebel, A., et al. 2008, A\&A, 484, 721

Clark, P. C., Glover, S. C. O., Smith, R. J., et al. 2011, Science, 331, 1040

Collet, R., Asplund, M., \& Trampedach, R. 2006, ApJ, 644, L121

de Ruyter, S., van Winckel, H., Dominik, C., Waters, L. B. F. M., \& Dejonghe, H. 2005, A\&A, 435, 161

Frebel, A., Aoki, W., Christlieb, N., et al. 2005, Nature, 434, 871

Frebel, A., Collet, R., Eriksson, K., Christlieb, N., \& Aoki, W. 2008, ApJ, 684, 588 
Friedemann, C., Guertler, J. \& Loewe, M. 1996, A\&AS, 117, 205

Freedman, W. L., Madore, B. F., Gibson, B. K., et al. 2001, ApJ, 553, 47

Freytag, B., Steffen, M., \& Dorch, B. 2002, Astron. Nachr., 323, 213

Freytag, B., Steffen, M., Wedemeyer-Böhm, S., \& Ludwig, H.-G. 2010, CO5BOLD User Manual,

http://www.astro.uu.se/ bf/co5bold_main.html

Freytag, B., Steffen, M., Ludwig, H.-G., et al. 2012, J. Comput. Phys., 231, 919

Gielen, C., van Winckel, H., Min, M., Waters, L. B. F. M., \& Lloyd Evans, T. 2008, A\&A, 490, 725

Giridhar, S., Lambert, D. L., Reddy, B. E., Gonzalez, G., \& Yong, D. 2005, ApJ, 627,432

González Hernández, J. I., Bonifacio, P., Ludwig, H.-G., et al. 2010, A\&A, 519, A46

Goswami, A., \& Prantzos, N. 2000, A\&A, 359, 191

Greif, T. H., Springel, V., White, S. D. M., et al. 2011, ApJ, 737, 75

Iwamoto, N., Umeda, H., Tominaga, N., Nomoto, K., \& Maeda, K. 2005, Science, 309,451

Joggerst, C. C., Almgren, A., Bell, J., et al. 2010, ApJ, 709, 11

Kaeufl, H.-U., Ballester, P., Biereichel, P., et al. 2004, Proc. SPIE, 5492, 1218

Klessen, R. S., Glover, S. C. O., \& Clark, P. C. 2012, MNRAS, 421, 3217

Kobayashi, C., \& Nakasato, N. 2011, ApJ, 729, 16

Korn, A. J., Richard, O., Mashonkina, L., et al. 2009, ApJ, 698, 410

Kurucz, R. L. 2005, Mem. Soc. Astron. It. Suppl., 8, 14

Lamers, H. J. G. L. M. 1986 A\&A, 159, 90

Lai, D. K., Bolte, M., Johnson, J. A., et al. 2008, ApJ, 681, 1524

Limongi, M., \& Chieffi, A. 2012, ApJS, 199, 38
Limongi, M., Chieffi, A., \& Bonifacio, P. 2003, ApJ, 594, L123

Lodders, K. 2003, ApJ, 591, 1220

Moriya, T., Tominaga, N., Tanaka, M., et al. 2010, ApJ, 719, 1445

Nissen, P. E., Akerman, C., Asplund, M., et al. 2007, A\&A, 469, 319

Pinsonneault, M. 1997, ARA\&A, 35, 557

Podobedova, L. I., Kelleher, D. E., \& Wiese, W. L. 2009, J. Phys. Chem. Ref. Data, 38, 171

Salvadori, S., Schneider, R., \& Ferrara, A. 2007, MNRAS, 381, 647

Sbordone, L., Bonifacio, P., Caffau, E., et al. 2010, A\&A, 522, A26

Schneider, R., Ferrara, A., Salvaterra, R., Omukai, K., \& Bromm, V. 2003, Nature, 422, 869

Schneider, R., Omukai, K., Bianchi, S., \& Valiante, R. 2012a, MNRAS, 419, 1566

Schneider, R., Omukai, K., Limongi, M., et al. 2012b, MNRAS, L444

Spite, M., Cayrel, R., Plez, B., et al. 2005, A\&A, 430, 655

Spite, M., Caffau, E., Andrievsky, S. M., et al. 2011, A\&A, 528, A9

Takeda, Y., Parthasarathy, M., Aoki, W., et al. 2002, PASJ, 54, 765

Takeda, Y., Hashimoto, O., Taguchi, H., et al. 2005, PASJ, 57, 751

Umeda, H., \& Nomoto, K. 2003, Nature, 422, 871

Van Winckel, H., Mathis, J. S., \& Waelkens, C. 1992, Nature, 356, 500

Venn, K. A., \& Lambert, D. L. 2008, ApJ, 677, 572

Waelkens, C., Van Winckel, H., Bogaert, E., \& Trams, N. R. 1991, A\&A, 251, 495

Wedemeyer, S., Freytag, B., Steffen, M., Ludwig, H.-G., \& Holweger, H. 2004 A\&A, 414, 1121

Zhang, W., Woosley, S. E., \& Heger, A. 2008, ApJ, 679, 639 